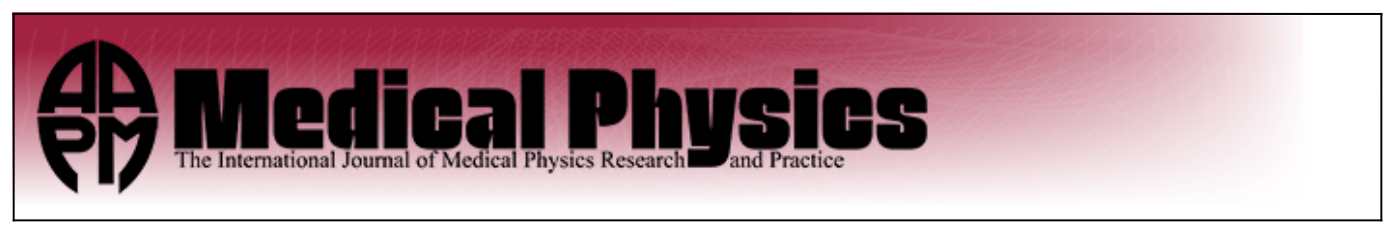

\title{
Assessment of dedicated low-dose cardiac micro-CT reconstruction algorithms using the left ventricular volume of small rodents as a performance measure
}

Joscha Maier, Stefan Sawall, and Marc Kachelrieß

Citation: Medical Physics 41, 051908 (2014); doi: 10.1118/1.4870983

View online: http://dx.doi.org/10.1118/1.4870983

View Table of Contents: http://scitation.aip.org/content/aapm/journal/medphys/41/5?ver=pdfcov

Published by the American Association of Physicists in Medicine

\section{Articles you may be interested in}

Deriving adaptive MRF coefficients from previous normal-dose CT scan for low-dose image reconstruction via penalized weighted least-squares minimization

Med. Phys. 41, 041916 (2014); 10.1118/1.4869160

Automatic quantification of epicardial fat volume on non-enhanced cardiac CT scans using a multi-atlas segmentation approach

Med. Phys. 40, 091910 (2013); 10.1118/1.4817577

Imaging of cardiac perfusion of free-breathing small animals using dynamic phase-correlated micro-CT

Med. Phys. 39, 7499 (2012); 10.1118/1.4762685

Temporal resolution improvement in cardiac CT using PICCS (TRI-PICCS): Performance studies

Med. Phys. 37, 4377 (2010); 10.1118/1.3460318

Dual-source spiral CT with pitch up to 3.2 and $75 \mathrm{~ms}$ temporal resolution: Image reconstruction and assessment of image quality

Med. Phys. 36, 5641 (2009); 10.1118/1.3259739

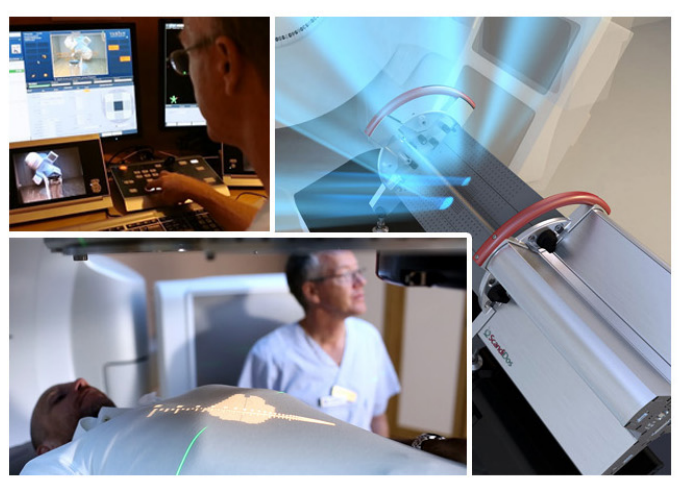

ScandiDos Delta 4 family

offers precise and easy

QA from plan to the last

fraction

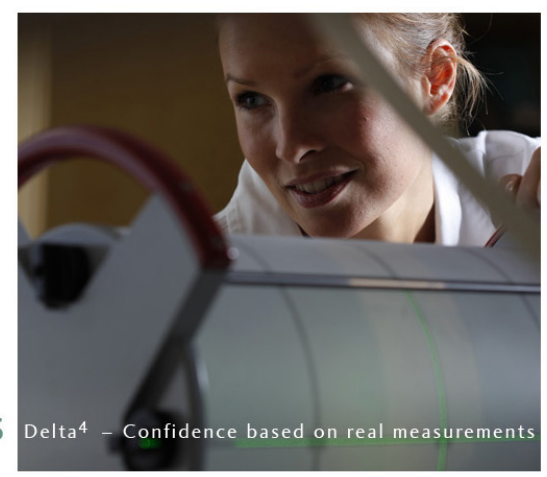




\title{
Assessment of dedicated low-dose cardiac micro-CT reconstruction algorithms using the left ventricular volume of small rodents as a performance measure
}

\author{
Joscha Maiera) \\ Medical Physics in Radiology, German Cancer Research Center (DKFZ), Im Neuenheimer Feld 280, \\ 69120 Heidelberg, Germany \\ Stefan Sawall and Marc Kachelrieß \\ Medical Physics in Radiology, German Cancer Research Center (DKFZ), Im Neuenheimer Feld 280, \\ 69120 Heidelberg, Germany and Institute of Medical Physics, University of Erlangen-Nürnberg, \\ 91052 Erlangen, Germany
}

(Received 21 August 2013; revised 26 March 2014; accepted for publication 30 March 2014; published 22 April 2014)

\begin{abstract}
Purpose: Phase-correlated microcomputed tomography (micro-CT) imaging plays an important role in the assessment of mouse models of cardiovascular diseases and the determination of functional parameters as the left ventricular volume. As the current gold standard, the phase-correlated Feldkamp reconstruction (PCF), shows poor performance in case of low dose scans, more sophisticated reconstruction algorithms have been proposed to enable low-dose imaging. In this study, the authors focus on the McKinnon-Bates (MKB) algorithm, the low dose phase-correlated (LDPC) reconstruction, and the high-dimensional total variation minimization reconstruction (HDTV) and investigate their potential to accurately determine the left ventricular volume at different dose levels from 50 to $500 \mathrm{mGy}$. The results were verified in phantom studies of a five-dimensional (5D) mathematical mouse phantom.

Methods: Micro-CT data of eight mice, each administered with an x-ray dose of $500 \mathrm{mGy}$, were acquired, retrospectively gated for cardiac and respiratory motion and reconstructed using PCF, MKB, LDPC, and HDTV. Dose levels down to $50 \mathrm{mGy}$ were simulated by using only a fraction of the projections. Contrast-to-noise ratio (CNR) was evaluated as a measure of image quality. Left ventricular volume was determined using different segmentation algorithms (Otsu, level sets, region growing). Forward projections of the 5D mouse phantom were performed to simulate a micro-CT scan. The simulated data were processed the same way as the real mouse data sets.

Results: Compared to the conventional PCF reconstruction, the MKB, LDPC, and HDTV algorithm yield images of increased quality in terms of CNR. While the MKB reconstruction only provides small improvements, a significant increase of the CNR is observed in LDPC and HDTV reconstructions. The phantom studies demonstrate that left ventricular volumes can be determined accurately at $500 \mathrm{mGy}$. For lower dose levels which were simulated for real mouse data sets, the HDTV algorithm shows the best performance. At $50 \mathrm{mGy}$, the deviation from the reference obtained at $500 \mathrm{mGy}$ were less than 4\%. Also the LDPC algorithm provides reasonable results with deviation less than $10 \%$ at $50 \mathrm{mGy}$ while PCF and MKB reconstruction show larger deviations even at higher dose levels.

Conclusions: LDPC and HDTV increase CNR and allow for quantitative evaluations even at dose levels as low as $50 \mathrm{mGy}$. The left ventricular volumes exemplarily illustrate that cardiac parameters can be accurately estimated at lowest dose levels if sophisticated algorithms are used. This allows to reduce dose by a factor of 10 compared to today's gold standard and opens new options for longitudinal studies of the heart. (C) 2014 American Association of Physicists in Medicine. [http://dx.doi.org/10.1118/1.4870983]
\end{abstract}

Key words: micro-CT, phase correlation, dose reduction, small animal imaging, left ventricular volume

\section{INTRODUCTION}

Mouse models play an important role in cardiovascular research. Several models have been established to investigate a variety of cardiovascular diseases such as myocardial infarction, ${ }^{1}$ dilated cardiomyopathy, ${ }^{2}$ or cardiac hypertrophy. ${ }^{3}$ Besides echocardiography ${ }^{4-7}$ and magnetic resonance imaging, ${ }^{8-11}$ microcomputed tomography (micro-CT) (Refs. 12-15) is a widely used imaging modality to assess morphological and functional parameters in these models, e.g., the left ventricular volume (LVV) or the ejection fraction. However, due to the high heart rates of mice of up to 600 beats per minute, cardiac micro-CT imaging is challenging. Cardiac motion, resulting from the heartbeat itself as well as induced by respiration, has to be considered in the data acquisition or in the reconstruction process to avoid the introduction of motion artifacts. ${ }^{12,16,17}$ Therefore, cardiac and respiratory gating signals which represent the corresponding motion states are 
required. According to the gating signals either the CT system is triggered to acquire projections only if the specimen is in a predefined motion state (prospective gating ${ }^{18}$ ) or the acquired signals are used to retrospectively sort the projections for a desired motion phase (retrospective gating ${ }^{17}$ ). Compared to retrospectively gated scans, prospective gating approaches are superior in terms of administered radiation dose as no redundant data in undesired motion states are acquired. However, as retrospective gating is technically less demanding and can be performed with most micro-CT systems without additional modifications, it is often the method of choice.

The current gold standard applied to such scans, the phasecorrelated Feldkamp (PCF) reconstruction, therefore, defines respiratory windows $\Delta r$ and cardiac windows $\Delta c$ that correspond to the motion state to be reconstructed. Depending on the desired temporal resolution, these windows are usually chosen between $5 \%$ and $20 \%$ of the respiratory or cardiac motion cycle, respectively. To finally obtain time resolved representations of the heart, the PCF reconstruction, filters and backprojects only the projections which were acquired within the predefined temporal window. Thus, in case of respiratory and cardiac gating, only $0.25 \%(\Delta c=5 \%, \Delta r=5 \%)$ to $4 \%$ ( $\Delta c=20 \%, \Delta r=20 \%)$ of the acquired projections are used for the reconstruction.

As a result, images may contain streak artifacts due to sparse and irregular angular sampling. Furthermore, the images comprise high noise levels unless high radiation doses of up to $1.5 \mathrm{~Gy}$ are administered. ${ }^{19}$ Although such dose levels are in general not lethal to mice, ${ }^{20}$ they may be sufficient to cause deterministic effects in the animal which could influence the outcome of the experiment. ${ }^{21}$ Especially for longterm studies which include numerous scans, the dose is an important issue and should be kept as low as reasonable achievable. As the conventional PCF reconstruction yields images of inacceptable quality in case of low dose scans, several efforts have been made to develop alternative reconstruction techniques that enable low dose imaging. The key idea of these approaches to overcome the problem of angular undersampled rawdata and noisy reconstructions is to incorporate prior knowledge into the reconstruction process.

Assuming that medical images consist of piecewise homogeneous structures, edge preserving image filters, or reconstruction techniques based on the compressed sensing theory can be used to address this issue. Sawall et al. ${ }^{22}$ introduced the low-dose phase-correlated (LDPC) reconstruction algorithm. It uses a McKinnon-Bates (MKB) prior to reduce streak artifacts and applies a five-dimensional bilateral filter, i.e., three spatial dimensions and two temporal dimensions for cardiac and respiratory motion that reduces noise in the final image. Sidky and Pan ${ }^{23}$ introduced the adaptive steepest descent projection onto convex sets (ASD-POCS) algorithm that iteratively reconstructs the image by minimizing the threedimensional total variation (TV) and enforcing constraints on rawdata fidelity. Its application to micro-CT data was presented by Han et al. ${ }^{24}$ As the gradient between reconstructions of adjacent respiratory or cardiac phases is also assumed to be sparse, the high-dimensional total variation (HDTV) minimization reconstruction proposed by Ritschl et al. ${ }^{25}$ extends the concept of total variation minimization to four dimensions to further improve image quality.

Besides the compressed sensing-based approaches, there are several approaches that aim at substituting missing data by measured data. Armitage et $a .^{26}$ introduced a least error projection sorting technique that replaces missing data by projections acquired in other motion phases according to a minimal temporal error parameter. Badea et al. ${ }^{27}$ tried to reduce streak artifacts by deconvolution in frequency space. Gaps in the Fourier transform of the PSF that arise due to sparse angular sampling are replaced by the corresponding information of a prior data set with appropriate angular sampling. Badea et al. ${ }^{28}$ acquired projection of one motion phase with adequate angular sampling while other motion phases were acquired with sparse angular sampling. To obtain high quality representations of every motion phase, the reconstruction of the adequately sampled rawdata is registered to the low quality reconstructions.

While today's clinical CT-systems already implement more sophisticated reconstruction algorithms to reduce radiation dose or to provide images of improved quality at the same dose level, such algorithms are rarely used in cardiac micro-CT imaging. Therefore, this paper exemplarily focuses on the MKB, the LDPC, and the HDTV algorithms and aims at demonstrating their benefit in terms of dose reduction compared to the conventional PCF reconstruction. It provides a quantitative evaluation of the achievable dose reduction based on real micro-CT mouse data sets as well as on simulated micro-CT data. Similar to Badea et al..$^{29}$ we use the left ventricular volume, an often used predictor of cardiovascular diseases, as performance measure.

\section{MATERIALS AND METHODS}

\section{A. Animal preparation}

For this study, cone-beam micro-CT scans of eight mice were performed. The mice were anesthetized with a combination of ketamine and rompun. As the contrast between the attenuation values of blood and myocardium is low, it has to be enhanced to ensure their distinction in the reconstructed images. Therefore, we administered an alkaline earth metal-based blood pool contrast agent (ExiTron nano 12000, Miltenyi Biotec $\mathrm{GmbH}$, Bergisch Gladbach, Germany and nanoPET Pharma GmbH, Berlin, Germany) providing a CT value of $12000 \mathrm{HU}$ in undiluted state and a prolonged blood circulation time of up to several hours. A volume of $100 \mu \mathrm{l}$ of the blood pool agent in combination with $20 \mu \mathrm{l} \mathrm{NaCl}$ chaser were injected in the tail vain over a time of $5 \mathrm{~min}$.

\section{B. Data acquisition}

The micro-CT scans were performed on a cone-beam micro-CT scanner (TomoScope Synergy Twin, CT Imaging $\mathrm{GmbH}$, Erlangen, Germany). The system consists of a microfocus $\mathrm{x}$-ray source mounted at a distance of $170 \mathrm{~mm}$ to the isocenter and a $1024 \times 1024$ flat detector mounted at a distance of $39 \mathrm{~mm}$ to the isocenter. The size of the quadratic 
detector pixels was $50 \mu \mathrm{m}$ with an effective pixel size of $40 \mu \mathrm{m}$ in the isocenter. To increase the detectors readout rate to 25 frames per second, a $2 \times 2$ binning of the detector pixels was performed leading to an effective pixel size of $80 \mu \mathrm{m}$ in the isocenter. The scans were conducted at $60 \mathrm{kV}$ tube voltage with a tube current of $0.3 \mathrm{~mA}$. Each scan consists of 7200 projections which were acquired continuously in a circular trajectory over an angular range of $3600^{\circ}$. The scan time for these ten rotations was $288 \mathrm{~s}$. The tube current time product was $87 \mathrm{mAs}$ and the absorbed dose was measured as $500 \mathrm{mGy}$ in each case using an ionization chamber (PTW-UNIDOS, PTW, Freiburg, Germany).

\section{C. Intrinsic gating}

To assign a certain cardiac and respiratory motion phase to every acquired projection, an intrinsic gating technique, also called kymogram, was used. This technique which obtains the phase information directly from the rawdata was first introduced to clinical spiral CT scanners by Kachelrieß et al. ${ }^{30}$ Whereas in clinical CT sophisticated mathematical models are required to account for fast gantry rotation ${ }^{30}$ (up to three rotations during one cardiac cycle), rather simple techniques can be applied to slowly rotating micro-CT scanners. In our case, the time of one gantry rotation is about two to three magnitudes longer than the time of a typical cardiac or respiratory cycle. Therefore, we followed an approach similar to Kuntz et $a l .{ }^{31}$ that evaluates the mean intensity inside a region of interest (ROI) in the acquired projections. In particular, this method works as follows. An initial reconstruction using all acquired projections is performed. A spherical ROI is defined in the reconstructed volume that covers parts of the moving organs, i.e., the heart or the lung. A forward projection of the ROI yields its position in the rawdata. For every acquired projection, the mean value inside the forward projected ROI is evaluated. As respiratory and cardiac motion lead to intensity changes in the acquired projections, the variation of the calculated mean is a surrogate signal for organ motion and can be used to generate gating signals. To further improve the signal quality, a bandpass filter was applied to extract the respiratory frequency or the cardiac frequency, respectively. Additional details are provided by Dinkel et al. ${ }^{32}$

\section{D. Reconstruction techniques}

\section{D.1. PCF reconstruction}

A standard reconstruction for projections acquired in cone-beam geometry is the Feldkamp-David-Kress algorithm (FDK) ${ }^{33}$ It filters (in our implementation with a RamLak kernel) and subsequently backprojects the rawdata to obtain a three-dimensional representation of the object $f_{\text {std }}$

$$
f_{\text {std }}=\mathrm{X}^{-1} p \text {. }
$$

Therein, $\mathrm{X}$ is the $\mathrm{x}$-ray transform operator and $p$ are the acquired projections. As the motion state of the object is not considered in this reconstruction, the image suffers from motion artifacts but exhibits a low noise level. To explicitly ac- count for organ motion, a phase-correlated version of this algorithm is used to overcome these issues

$$
f_{\mathrm{PCF}}=\mathrm{X}_{\mathrm{PC}}^{-1} p \text {. }
$$

$\mathrm{X}_{\mathrm{PC}}$ denotes the phase-correlated $\mathrm{x}$-ray transform that only backprojects projections that were acquired within a distinct respiratory window centered at phase $r$ and a cardiac window centered at phase $c$. The widths of these windows $\Delta r$ and $\Delta c$, are usually chosen to be between $5 \%$ and $20 \%$, depending on the desired image quality and the integration time of the detector. Therefore, the PCF reconstruction uses only $0.25 \%$ $(\Delta r=5 \%, \Delta c=5 \%)$ to $4 \%(\Delta r=20 \%, \Delta c=20 \%)$ of the total amount of acquired projections for the reconstruction of a single volume. As a result of the reduced number of projections and the irregular angular sampling, the reconstructions may suffer from streak artifacts and increased noise levels. Especially if the radiation dose is decreased this becomes apparent as only a very limited number of projections might be provided.

\section{D.2. MKB algorithm}

The McKinnon-Bates algorithm was introduced by McKinnon and Bates ${ }^{34}$ to reduce streak artifacts in the PCF reconstructions of the human heart. Garden and Robb ${ }^{35}$ provided a practical implementation. The idea of this algorithm is to add an image $f_{\text {corr }}$ to the PCF reconstruction that corrects for the streak artifacts. To obtain the correction image, a forward projection of a standard reconstruction as given by Eq. (1) is performed. A phase-correlated reconstruction of the forward projected rawdata yields the standard image with similar streak artifacts as the PCF reconstruction. The final correction image that exhibits only the streak artifacts can be received by subtracting this image of the standard reconstruction. Mathematically, the resulting McKinnon-Bates image $f_{\mathrm{MKB}}$ is given by

$$
\begin{aligned}
f_{\mathrm{MKB}} & =f_{\mathrm{PCF}}+f_{\text {corr }} \\
& =f_{\mathrm{PCF}}+\left(f_{\text {std }}-\mathrm{X}_{\mathrm{PC}}^{-1}\left(\mathbf{X} f_{\text {std }}\right)\right) \\
& =\mathrm{X}_{\mathrm{PC}}^{-1} p+\left(f_{\text {std }}-\mathbf{X}_{\mathrm{PC}}^{-1}\left(\mathbf{X} f_{\text {std }}\right)\right) .
\end{aligned}
$$

The drawback of the McKinnon-Bates algorithm is that image noise is increased due to the addition of the correction image.

\section{D.3. LDPC reconstruction}

The LDPC algorithm was proposed by Sawall et al. ${ }^{22} \mathrm{It}$ uses a MKB reconstruction that reduces streak artifacts as prior and applies a bilateral filter in up to five dimensions to achieve low noise levels while preserving edges. To account for respiratory and cardiac motion, the MKB approach is performed as a two step algorithm. In a first step, the MKB algorithm is only applied with respect to respiratory motion, in a second step the respiratory gated reconstruction is used as prior for additional cardiac gating. This yields fivedimensional volumes $f(x, y, z, r, c)$, where $x, y$, and $z$ denote the three spatial dimensions and $r$ and $c$ denote the cardiactemporal dimension and the respiratory-temporal dimension. 
The bilateral filter applied to these volumes is defined as follows:

$$
\mathrm{B}_{5 \mathrm{D}} f(\boldsymbol{x})=\frac{\int d^{5} x^{\prime} \mathrm{D}\left(\boldsymbol{x}, \boldsymbol{x}^{\prime}\right) \mathrm{R}\left(\boldsymbol{x}, \boldsymbol{x}^{\prime}\right) f\left(\boldsymbol{x}^{\prime}\right)}{\int d^{5} x^{\prime} \mathrm{D}\left(\boldsymbol{x}, \boldsymbol{x}^{\prime}\right) \mathrm{R}\left(\boldsymbol{x}, \boldsymbol{x}^{\prime}\right)}
$$

where $\mathrm{D}$ denotes the domain filter controlling the spatial extent of the filter and $\mathrm{R}$ denotes the range filter regulating the contribution of pixels located at positions $\boldsymbol{x}^{\prime}$ with respect to the gray level difference $f(\boldsymbol{x})-f\left(\boldsymbol{x}^{\prime}\right)$.

According to Sawall et al. ${ }^{22}$ the range filter is given by a one-dimensional Gaussian with standard deviation $\sigma_{f}$, while the domain filter is represented by a five-dimensional Gaussian with varying standard deviations $\sigma_{x}, \sigma_{y}, \sigma_{z}, \sigma_{r}$, and $\sigma_{c}$ for each dimension.

A three-dimensional and a four-dimensional (4D) bilateral filter was also applied to the first prior volume $f(x, y, z)$ and the second prior volume $f(x, y, z, r)$ using the same filter parameters as for the final five-dimensional filtering. An extensive evaluation of this method is provided by Sawall et al. ${ }^{22}$

\section{D.4. HDTV minimization reconstruction}

HDTV is an iterative algorithm proposed by Ritschl et al. $^{25}$ that is based on the compressed sensing theory. Such algorithms try to solve

$$
\min \|\Psi(f)\|_{1} \text { subject to }\|\mathbf{X} f-p\|_{2}^{2}<\epsilon,
$$

where $\Psi$ is a sparsifying transform applied to the image $f$. In clinical CT as well as micro-CT, the images are assumed to consist of piecewise homogenous structures, and therefore, the image gradient turns out to be an adequate sparsifying transform. Including a priori knowledge in terms of gradient sparseness leads to a TV prior. ${ }^{36}$ Several groups have proposed a three-dimensional TV regularization for iterative reconstructions. $^{23,37-39}$ In double-gated micro-CT imaging, we deal with five-dimensional volumes $f(r, c, x, y, z)$. As images of neighboring phases are similar in regions without organ motion, the corresponding temporal gradient can also be assumed to be sparse. That enables the extension of the TV regularization to up to five dimensions which is referred to as HDTV.

To reduce computational time and memory requirements, the TV was only extended to four dimensions:

The three spatial dimensions as well as the respiratorytemporal dimension.

This leads to the following minimization problem:

$$
\min \|\operatorname{HDTV}(f)\|_{1} \text { subject to }\|\mathrm{X} f-p\|_{2}^{2}<\epsilon \text {. }
$$

To minimize this cost functional, both terms of Eq. (6) are treated separately. The data fidelity term is minimized by an ordered subsets simultaneous algebraic reconstruction technique (OSSART) (Ref. 40)

$$
f_{n+1}=f_{n}+\beta \frac{1}{\mathrm{X}_{\mathrm{PC}}^{\mathrm{T}} \mathbb{1}} \mathrm{X}_{\mathrm{PC}}^{\mathrm{T}} \frac{\mathrm{X}_{\mathrm{PC}} f_{n}-p_{\mathrm{PC}}}{\mathrm{X}_{\mathrm{PC}} \mathbb{1}} .
$$

Each OSSART update step is followed by a 4D TV minimization using a gradient descent method

$$
f_{n+1, m+1}=f_{n+1, m}-\alpha \cdot \nabla_{f_{n+1, m}}\left[\operatorname{HDTV}\left(f_{n+1, m}\right)\right] .
$$

For an extensive description, we refer to Ritschl et al. ${ }^{25}$

\section{E. Segmentation}

A semiautomatic approach was applied to segment the left ventricle in the reconstructed volumes. In a first step, a threedimensional ROI that contains the left ventricle was created manually as shown in Fig. 1. For every mouse and every cardiac motion state, the ROI was defined to have its surface inside the myocardium except at the valve plane. In a second step, the voxels within that ROI were sorted into two classes: blood/contrast agent and myocardium.

In order to demonstrate that the segmentation results are not only valid for a certain type of segmentation algorithm, three different algorithms which are commonly used for medical image segmentation, were applied: A histogram-based, a region-based, and a level sets algorithm. Histogram-based algorithms only consider the histogram data of the image to be segmented and try to find a set of thresholds that divides the histogram into classes with respect to a certain cost function. For our purpose, we used the Otsu algorithm to find the ideal threshold. ${ }^{41}$ It defines a statistical measure, the so called interclass variance that indicates how good a set of $k$ thresholds $T_{k}$ separates the image histogram into $k+1$ classes. A maximization of this quantity finally yields the optimal threshold. As the calculation of a histogram drastically reduces the amount of data to be processed, such algorithms show a high computational performance and therefore, find various applications. A further speed up can be achieved by using lookup tables within the optimization process as introduced by Liao et $a l .{ }^{42}$ However, since the information of the spatial distribution of gray levels is lost in the histogram data, the segmentation results may not be physiological, especially in case of high noise levels. Therefore, region-based segmentation approaches incorporate prior knowledge on the image as the connectivity of the two regions to be segmented. Representative for such approaches, we used a seeded region growing algorithm. ${ }^{43}$ It requires a manual definition of two initial seed regions that correspond to the left ventricle and the myocardium. Subsequently, these regions are grown in an iterative manner until all voxels are assigned to one of the predefined regions. In each iteration, the voxel whose gray level has the smallest difference to the mean gray level of the region it

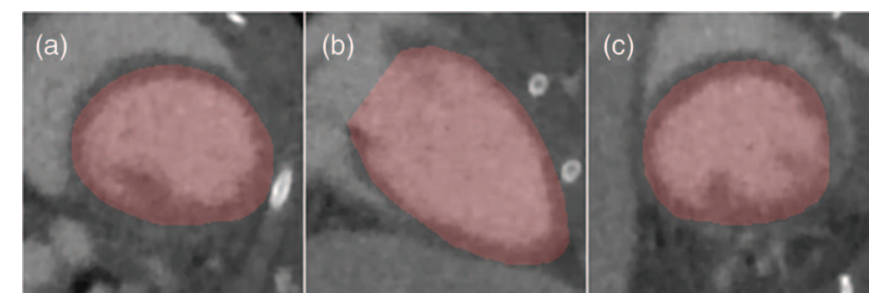

FIG. 1. Reconstruction of the heart overlayed with a typical ROI in an axial (a), a coronal (b) and a sagittal (c) plane. 
adjoins is added to that region. This process assures that the final segmentation result are two connected regions. Finally, a level sets segmentation algorithm as proposed by Osher and Sethian ${ }^{44}$ was applied. It provides a numerical technique to track $N$-dimensional surfaces on a Cartesian grid which can be applied to image segmentation. Therefore, an initial surface is defined within the object and evolved to converge at its boundaries. In this process, the evolution of the curve is performed with respect to a so-called speed function which provides the direction and the magnitude of the curve evolution. It is designed to approach zero at the object boundaries and can include morphological constraints according to the segmentation problem. For our purpose, we used a speed function as proposed by Chan and Vese ${ }^{45}$ which consists of a data-dependent as well as a curvature dependent term. Using a certain speed function leads to a set of partial differential equations which can be solved numerically, e.g., with the finite element method. However, as solving differential equations is computationally expensive, we followed the approach of Shi and $\mathrm{Karl}^{46}$ that realizes the surface evolution with a switching mechanism between two linked lists.

\section{F. Mouse phantom simulations}

As the in vivo mouse data sets do not allow for the computation of a ground truth, we designed a mathematical, fivedimensional mouse phantom to evaluate the accuracy of the estimated left ventricular volumes (see Fig. 2).

Our aim was to derive projection images from a voxel phantom that imitates the heart beat of a real mouse. For our purpose, it is based on a standard reconstruction of a $500 \mathrm{mGy}$ scan which was segmented, using a multilevel Otsu algorithm, to contain 15 different gray levels. The static and blurred representation of the left ventricle of the prior was removed by setting its gray levels within the ROI (see Sec. 2.E and Fig. 2) to the ones of the myocardium. Subsequently, x-ray projec- tions were simulated by a forward projection of the phantom using the geometry of our micro-CT system. Prior to every forward projection a left ventricle with known volume was inserted in place of the removed ventricle. The ventricle inserts were generated as follows: First, LDPC reconstructions of the same mouse data set were performed for systolic and diastolic cardiac motion state as well as end-inhale and endexhale respiratory motion state. As these motion states define the maxima and minima of the cardiac and respiratory cycle, we assumed that arbitrary motion states are between these extremal states. To derive them, the left ventricle was segmented in the LDPC reconstructions according to Sec. 2.E. Subsequently, the binary representations of the left ventricle in systole and diastole were added and filtered with a running mean filter to receive a smooth transition between gray levels. The application of a time-dependent threshold

$$
T_{c}(t)=1+0.5 \cdot \cos (2 \pi \cdot C(t)),
$$

which was determined with respect to the acquired cardiac gating signals $C(t)$, yields the binary representation of a motion state between systole and diastole. In order to consider the influence of respiration in this model, such a binary representation was derived for the end-inhale and end-exhale respiratory motion state. Similar to the cardiac case, the binary images were added and filtered with a running mean filter and a time-dependent threshold

$$
T_{r}(t)=1+0.5 \cdot \cos (2 \pi \cdot R(t)) \text {, }
$$

was applied. Here, $R(t)$ refers to the acquired respiratory gating signal. As we only focus on the left ventricle, the motion of the chest and other organs, which is induced by respiration, was neglected. As the prior as well as the left ventricle were derived from the same mouse data set, the insert fits into the prior without further modifications. Therefore, an addition of the binary left ventricle to the prior yields the final voxel phantom for a given projection.

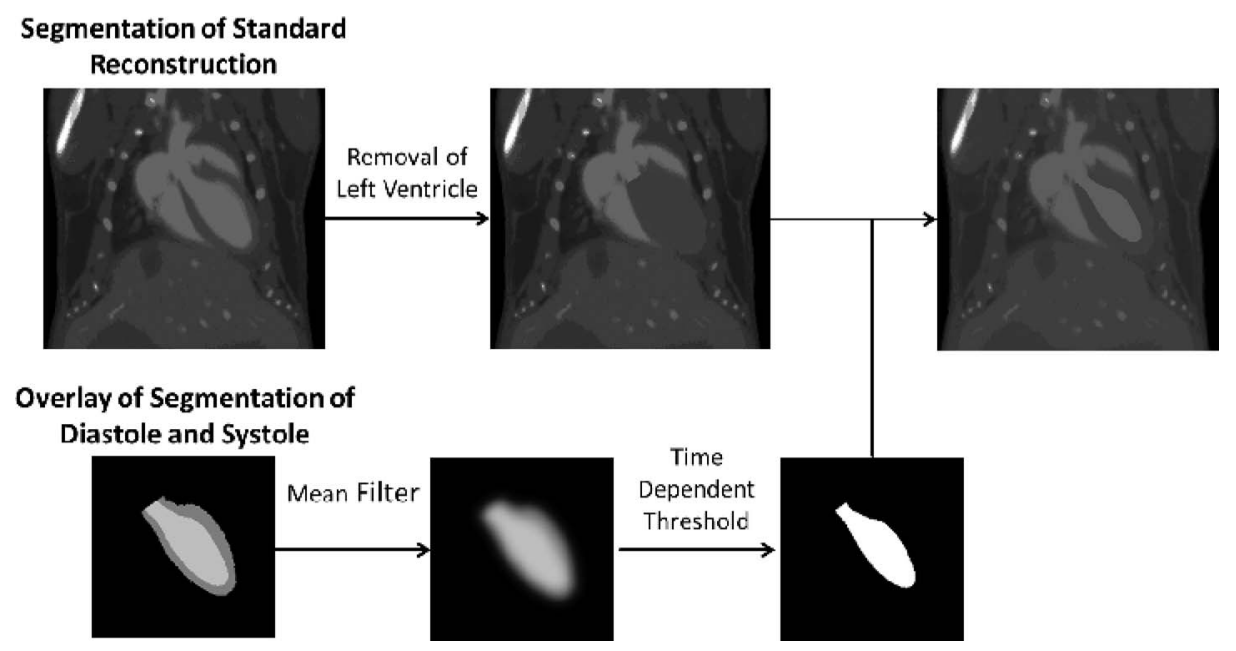

FIG. 2. Schematic of the generation of the voxel phantom for a certain motion state. A standard reconstruction of a 500 mGy scan which is segmented to contain 15 different gray levels is used as prior for the phantom. Subsequently, the left ventricle is removed by setting its gray levels equal to the gray levels of the myocardium. A sufficient insert that represents the left ventricle in the motion state to be simulated is derived as follows: First, the left ventricle in systole and diastole is segmented from a LDPC reconstruction. These binary representations of the left ventricle are then added and filtered with a running mean filter. Finally, a time-dependent threshold that corresponds to the desired motion state is applied and the resulting left ventricle is added to the prior. 


\section{RESULTS}

PCF, MKB, LDPC, and HDTV reconstructions were performed for eight micro-CT mouse data sets. For the acquisition of each data set, a total radiation dose of $500 \mathrm{mGy}$ was administered. Lower dose levels down to $50 \mathrm{mGy}$ were simulated retrospectively by only using the fraction of the first projections that corresponds to that dose level.

For every dose level, the respiratory as well as the cardiac cycle was divided into ten phases, as we expected that this allows for the estimation of the diastolic and systolic motion state with sufficient accuracy. Thus, the widths of the respiratory as well as the cardiac windows have to be at least $10 \%$ to cover the whole respiratory and cardiac cycle. However, we felt that choosing the respiratory window to be $15 \%$ of the respiratory cycle and the cardiac window to be $20 \%$ of the cardiac cycle is a reasonable trade-off between temporal resolution and image quality. This leads to a temporal overlap of the $5 \%$ for the respiratory phases and to one of $10 \%$ for the cardiac phases.

The isotropic voxel size was chosen as $60 \mu \mathrm{m}$. The PCF and the MKB reconstruction do not require additional reconstruction parameters. For the LDPC, the parameters $\sigma_{x}$, $\sigma_{y}$, and $\sigma_{z}$ used in the bilateral filter were set to $150 \mu \mathrm{m}$. The parameters $\sigma_{r}$ and $\sigma_{c}$ were set to $3 \Delta r$ and $\Delta c$, respectively. The standard deviation of the range filter $\sigma_{f}$ was set to a value slightly above the noise level of the MKB reconstruction. To receive sharp reconstructions, this value should not exceed the contrast between left ventricle and myocardium. The HDTV reconstruction was performed with 15 iterations, each consisting of one OSSART step including eight subiterations and one 4D TV minimization step that contains 20 iterations. The simulated phantom data were processed the same way. Exemplary reconstructions of one of the real mouse data sets, performed with these parameters are shown in Fig. 3 for the diastole and in Fig. 4 for the systole. Compared to the conventional PCF reconstruction the MKB, LDPC, and HDTV algorithms provide images of increased quality. While the MKB algorithm is only able to reduce streak artifacts within the reconstruction, the images obtained with the LDPC and the HDTV algorithm also reduce image noise substantially. Even at radiation doses as low as $50 \mathrm{mGy}$ the left ventricle is clearly distinguishable. In order to provide a quantitative measure of dose-dependent image quality, the contrast-to-noise ratio (CNR) is evaluated in Sec. 3.A. As we want to demonstrate that sophisticated reconstruction algorithms like LDPC and HDTV are able to determine the left ventricular volume at decreased dose levels we validated their accuracy in phantom studies (see Sec. 3.B). The evaluation of the real mouse data sets is given in Sec. 3.C.

\section{A. Evaluation of CNR}

As we focus on left ventricular volume estimation the contrast between the left ventricle and the myocardium was evaluated. Therefore, a three-dimensional ROI was defined within a homogenous region inside the left ventricle $\left(\mathrm{ROI}_{\mathrm{LV}}\right)$ and the

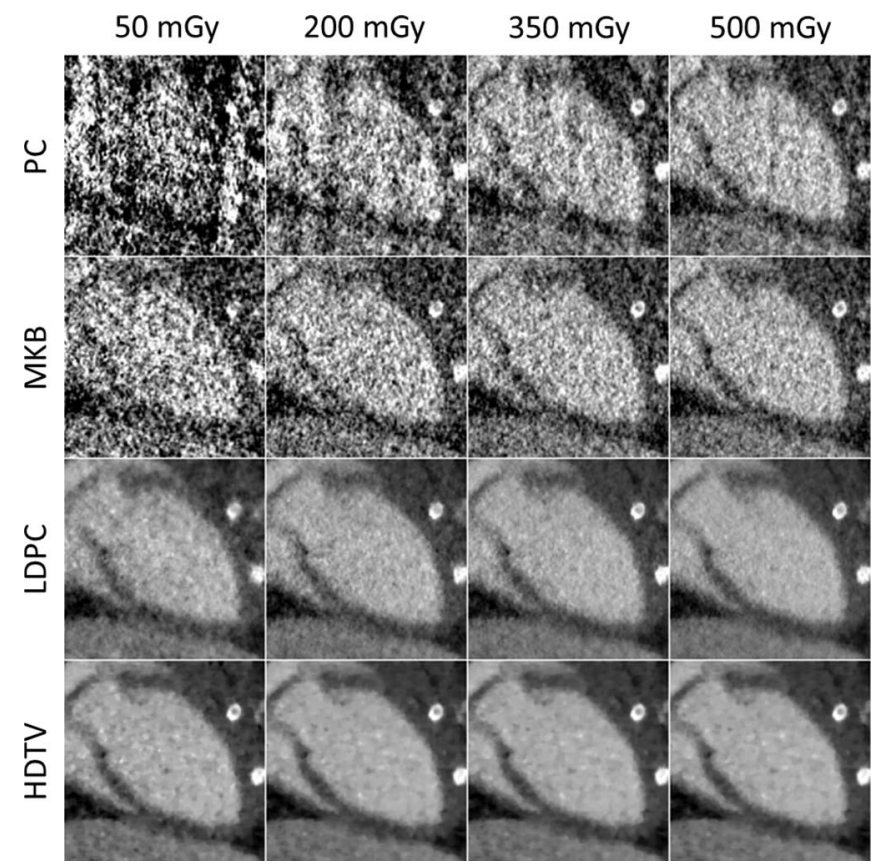

FIG. 3. Exemplary PCF, MKB, LDPC, and HDTV reconstruction of one of the real mouse data sets showing the left ventricle in the diastole for dose levels of 50, 200, 350, $500 \mathrm{mGy}(\mathrm{C}=500 \mathrm{HU} / \mathrm{W}=1300 \mathrm{HU})$.

myocardium ( $\left.\mathrm{ROI}_{\text {myocard }}\right)$, respectively. The CNR was calculated according to

$$
\mathrm{CNR}=\frac{\text { mean }\left(\mathrm{ROI}_{\mathrm{LV}}\right)-\text { mean }\left(\mathrm{ROI}_{\text {myocard }}\right)}{\sqrt{\left(\sigma\left(\mathrm{ROI}_{\mathrm{LV}}\right)^{2}+\sigma\left(\mathrm{ROI}_{\text {myocard }}\right)^{2}\right.}} .
$$

This definition also includes variations caused by streak artifacts, which could be avoided by evaluating the noise within

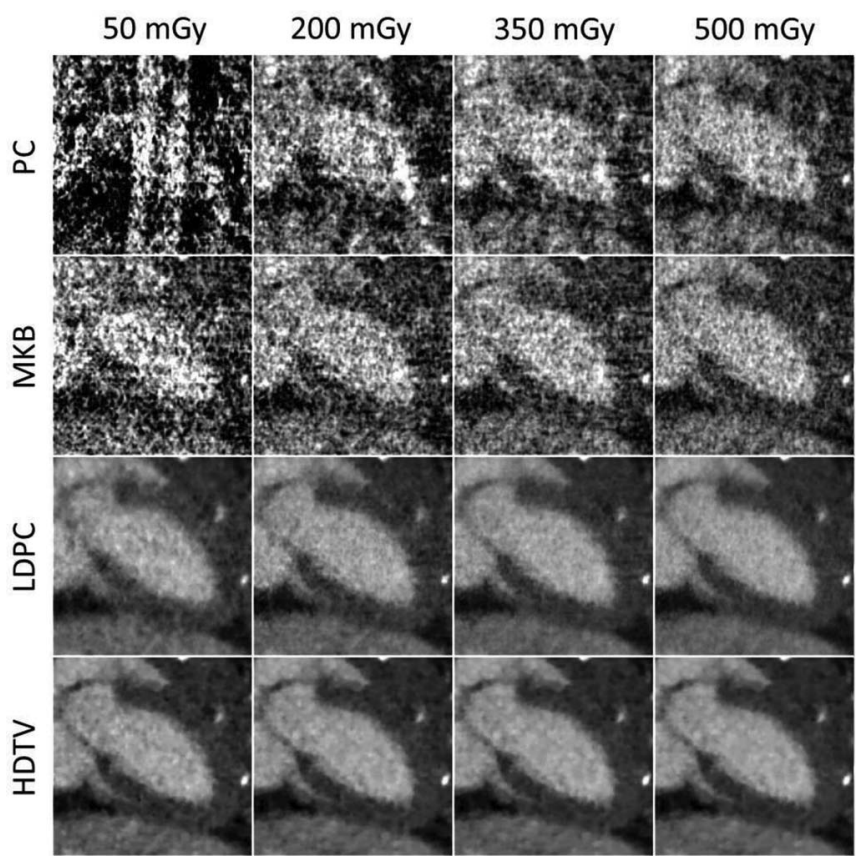

FIG. 4. Exemplary PCF, MKB, LDPC, and HDTV reconstruction of one of the real mouse data sets showing the left ventricle in the systole for dose levels of 50, 200, 350, $500 \mathrm{mGy}(\mathrm{C}=500 \mathrm{HU} / \mathrm{W}=1300 \mathrm{HU})$. 


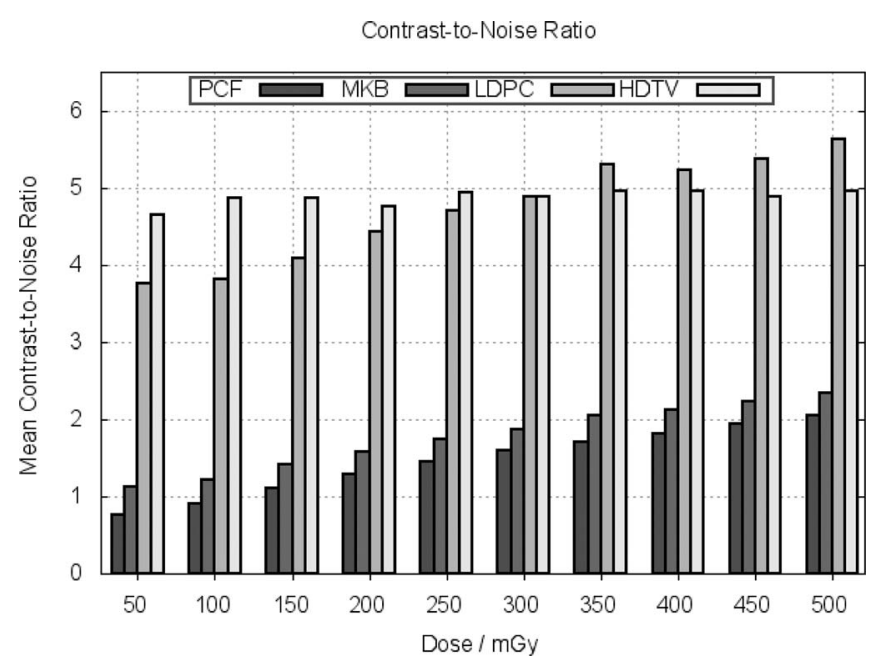

FIG. 5. Mean CNR evaluated in diastolic and systolic PC, MKB, LDPC, and HDTV reconstructions of eight mouse data sets.

a difference image of adjacent slices of the reconstructed volumes. However, we feel that the used approach represents the visual impression of image quality. For every mouse data set and every dose level, the CNR was computed in a reconstruction of the diastole and a reconstruction of the systole. The mean CNR of these evaluations is shown in Fig. 5. As the conventional PCF reconstruction suffers from high noise levels and streak artifacts, it shows the smallest CNR of about 2 at $500 \mathrm{mGy}$. For decreasing dose levels, the CNR evaluated in the PCF reconstructions declines continuously down to 0.8 at $50 \mathrm{mGy}$. Due to reduced streak artifacts, the results of the MKB algorithm show a slightly higher CNR compared to the PCF reconstruction. The LDPC and the HDTV algorithms which are designed to provide images with low noise levels and reduced artifacts show the best CNR. At $500 \mathrm{mGy}$, it is higher than in PCF and MKB reconstructions by a factor of 2-2.5. Even at dose levels of $50 \mathrm{mGy}$ LDPC and HDTV result in images of high quality with mean CNRs of 3.8 and 4.6, respectively. While the CNR of the HDTV reconstructions is almost constant for decreasing levels of radiation dose, the ability of the LDPC algorithm to reduce noise is limited by the CNR in the MKB image. The width of the range filter $\sigma_{f}$ has to be lower than the contrast to be resolved in order to preserve edges and thus cannot be adjusted arbitrarily to increased image noise. Therefore, the performance of the LDPC decreases for lower radiation doses.

\section{B. Phantom simulations}

To verify that functional parameters as left ventricular volume can be assessed accurately, we performed phantom studies based on the five-dimensional mouse phantom with known left ventricular volume as described in Sec. 2.F. A $500 \mathrm{mGy}$ scan was simulated using the acquisition parameters of our micro-CT system. These projections were reconstructed with PCF, MKB, LDPC, and HDTV using the same parameters as for the mouse data sets. To determine the left ventricular volume Otsu, seeded region growing and levels sets segmentation was applied to the reconstructions. The ground truth
TABLE I. Mean ventricular volume of the ground truth in systole and diastole as well as the mean relative deviation of the ground truth volume at $500 \mathrm{mGy}$.

\begin{tabular}{lllllll}
\hline \hline & & \multicolumn{4}{c}{ Deviation of ground truth } \\
\cline { 3 - 7 } & & Ground truth & PCF & MKB & LDPC & HDTV \\
\hline \multirow{2}{*}{ Otsu } & Systole & $27.29 \mu 1$ & $4.86 \%$ & $2.33 \%$ & $2.52 \%$ & $1.93 \%$ \\
& Diastole & $61.81 \mu 1$ & $1.86 \%$ & $1.48 \%$ & $1.08 \%$ & $0.77 \%$ \\
\hline \multirow{2}{*}{ Region growing } & Systole & $26.70 \mu 1$ & $1.60 \%$ & $1.45 \%$ & $1.30 \%$ & $1.48 \%$ \\
& Diastole & $62.33 \mu 1$ & $0.54 \%$ & $0.46 \%$ & $0.38 \%$ & $0.43 \%$ \\
\hline \multirow{2}{*}{ Level sets } & Systole & $26.93 \mu 1$ & $2.10 \%$ & $3.05 \%$ & $2.20 \%$ & $1.40 \%$ \\
& Diastole & $62.17 \mu 1$ & $2.33 \%$ & $2.10 \%$ & $1.47 \%$ & $1.13 \%$ \\
\hline \hline
\end{tabular}

was generated by simulating a noiseless scan with appropriate angular sampling. A PCF reconstruction was used to obtain an ideal reconstruction. A segmentation of this reconstruction with the corresponding segmentation algorithm yields the ground truth. All respiratory phases within diastolic and systolic cardiac phase were reconstructed and segmented separately. Table I shows the mean left ventricular volumes of the ground truth in systole and diastole as well as the relative deviation of the PCF, MKB, LDPC, and HDTV reconstructions from the ground truth at $500 \mathrm{mGy}$. The results indicate that at a dose level of $500 \mathrm{mGy}$ all reconstruction algorithms are able to provide images that allow for the accurate determination of the left ventricular volume. Except for the Otsu segmentation of the PCF reconstruction in the systole the deviation is not larger than $3 \%$. For the HDTV algorithm, the deviations are actually always less than $2 \%$.

It has to be noted that we used gating signals of real mouse scans for the simulation. As a result, the projections are not distributed equally in systole and diastole. For the used gating signals, there are slightly more projections acquired in the diastole than in the systole which leads to better image quality of reconstructions of the diastole and therefore, also more accurate segmentation results.

\section{C. Mouse data sets}

For dose levels between 50 and $500 \mathrm{mGy}$, reconstructions were performed using the PCF, MKB, LDPC, and the HDTV algorithm. The left ventricle was segmented in the reconstructions by Otsu, seeded region growing, and level sets segmentation. As the phantom studies demonstrated that at $500 \mathrm{mGy}$ all reconstruction algorithms provide images of which the left ventricular volume can be determined accurately, the $500 \mathrm{mGy}$ scan was used as as reference. The left ventricular volumes determined at lower dose levels were normalized to the volume determined at $500 \mathrm{mGy}$ to investigate the ability of the PCF, MKB, LDPC, and HDTV algorithms to reproduce this volume at lower dose levels. The corresponding evaluation of the mouse data sets are shown in Fig. 6. All segmentation algorithms show similar courses for decreasing radiation dose levels. Only Otsu's method seems to provide slightly smaller deviations for lowest dose levels. As it is a histogrambased approach, the resulting left ventricular volume is less sensitive to high image noise levels in case of bilevel 

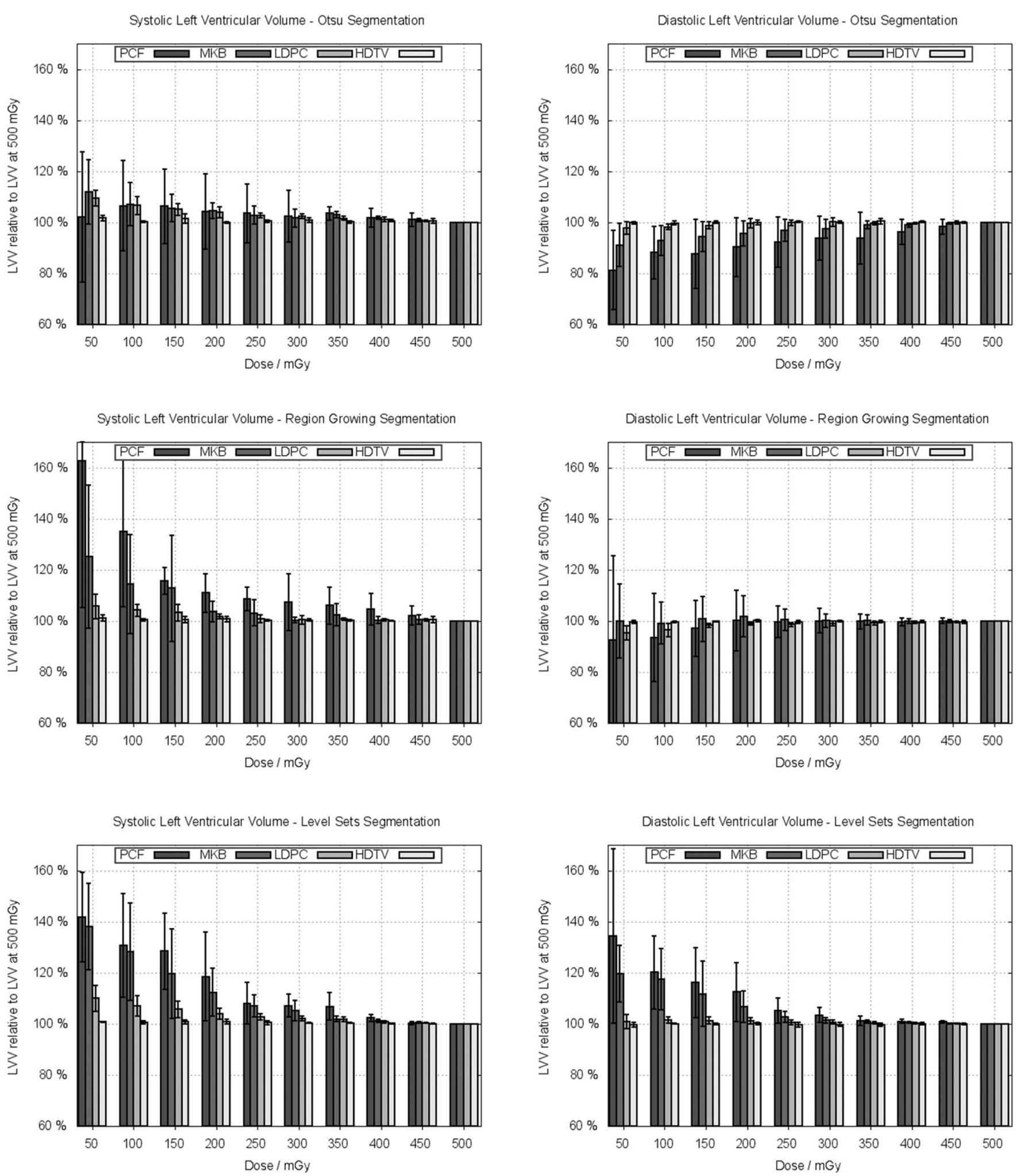

FIG. 6. Mean LVV and corresponding standard deviation of eight mice. For each mouse, the LVV was determined in a PC, MKB, LDPC, and HDTV reconstruction with Otsu (top row), seeded region growing (middle row), and level set (bottom row) segmentation. For every reconstruction, the LVV is normalized to its value at $500 \mathrm{mGy}$.

segmentation. However, it has to be noted that although the left ventricular volume might be accurate, the segmentation result can be unphysiological for images with low CNR, especially if the segmentation algorithm does not include morphological constraints as curvature or connectivity.
Considering the different reconstruction algorithms, the results demonstrate the poor performance of the conventional PCF reconstruction in case of low dose reductions. As the amount of streak artifacts as well as the image noise levels increase if the radiation dose is reduced, deviations larger 
than $10 \%$ can already be observed at dose levels of $350 \mathrm{mGy}$. Compared to the PCF reconstruction also rather simple algorithms as the MKB algorithm show considerable improvements. Down to a radiation dose level of $250 \mathrm{mGy}$ the reference left ventricular volume could be reproduced with an accuracy better than $10 \%$. More sophisticated algorithms as LDPC and HDTV show even better results at lower dose levels. While for the LDPC algorithm the deviations are less than $5 \%$ at $250 \mathrm{mGy}$ the HDTV algorithm actually yields images at $50 \mathrm{mGy}$ of which the reference left ventricular volume can be determined with a deviation less than $5 \%$.

\section{DISCUSSION AND CONCLUSION}

In this study, we investigated the ability of the PCF, MKB, LDPC, and HDTV algorithms to provide images that allow for the accurate determination of the left ventricular volume at dose levels between 50 and $500 \mathrm{mGy}$ using the micro-CT system described in Sec. 2.B. Based on phantom studies we demonstrated that all algorithms provide accurate left ventricular volumes at $500 \mathrm{mGy}$ which is a typical administered dose level in micro-CT studies. Evaluating the real mouse data sets, the 500 mGy scan was used as reference. Table II summarizes the minimal required dose to reproduce the reference left ventricular volume. If a deviation of less than $5 \%$ is demanded, the minimal administered dose of the PCF reconstruction that is required is $400-450 \mathrm{mGy}$, depending on the considered segmentation algorithm. If instead of the conventional PCF reconstruction the MKB, the LDPC, or the HDTV algorithm is used, the same accuracy can be provided at reduced dose levels. The achievable dose reductions are $11 \%-$ $25 \%$ for the MKB algorithm, 38\%-56\% for the LDPC algorithm, and more than $88 \%$ for the HDTV algorithm. As the image quality of the PCF reconstructions decreases faster for declining dose levels than the image quality provided by the MKB, LDPC, and HDTV reconstructions, the achievable dose reductions are even larger if the study only demands deviations less than $10 \%$. In that case, the MKB algorithm yields $43 \%-50 \%$ dose reduction, the LDPC algorithm $75 \%-$ $88 \%$ dose reduction, and the HDTV algorithm more than $86 \%$ dose reduction.

TABLE II. Minimal radiation dose level that is required to reproduce the left ventricular volume determined at $500 \mathrm{mGy}$ with a deviation less than $5 \%$ and $10 \%$.

\begin{tabular}{llllll}
\hline \hline & & \multicolumn{3}{c}{ Minimal radiation dose level } \\
\cline { 3 - 6 } & & $\begin{array}{c}\text { PCF } \\
(\mathrm{mGy})\end{array}$ & $\begin{array}{c}\text { MKB } \\
(\mathrm{mGy})\end{array}$ & $\begin{array}{l}\text { LDPC } \\
(\mathrm{mGy})\end{array}$ & $\begin{array}{c}\text { HDTV } \\
(\mathrm{mGy})\end{array}$ \\
\hline Deviation less & Otsu & 450 & 350 & 250 & $<50$ \\
than 5\% & Region growing & 450 & 400 & 200 & $<50$ \\
& Level sets & 400 & 300 & 250 & $<50$ \\
\hline Deviation less & Otsu & 400 & 200 & 200 & $<50$ \\
than 10\% & Region growing & 400 & 200 & $<50$ & $<50$ \\
& Level sets & 350 & 200 & 150 & $<50$ \\
\hline \hline
\end{tabular}

These evaluations demonstrate that quantitative evaluations of functional parameters in mouse models as the left ventricular volume can be determined accurately at dose levels as low as $50 \mathrm{mGy}$ if sophisticated reconstruction algorithms like the HDTV algorithm are used.

However, due to its iterative nature the HDTV algorithm is computationally expensive. In contrast to the conventional PCF reconstruction which requires only a single filtered backprojection, it requires several forward and backprojections as well as additional HDTV minimization steps. As we used 15 OSSART updates, each followed by a HDTV minimization, the computational effort is about 40 times higher compared to the PCF reconstruction. If computational speed is an issue, the LDPC algorithm provides a fast alternative. As it uses a MKB prior which requires one forward and two backprojections and finally applies a five-dimensional bilateral filter, its computational effort is about five times higher than the one of the PCF reconstruction. Despite comparably small reconstruction time requirements, the LDPC algorithm yields highly accurate results down to $200 \mathrm{mGy}$.

Due to the potential of the LDPC and especially the HDTV algorithm to reduce the administered radiation dose significantly compared to today's gold standard, these algorithms open new options for longitudinal studies of the heart.

\section{ACKNOWLEDGMENTS}

The high-speed image reconstruction software RayConStructIR was provided by RayConStruct GmbH, Nürnberg, Germany. The authors thank Sandra Strobelt and Andreas Hess, Institute of Experimental and Clinical Pharmacology and Toxicology, University of Erlangen-Nürnberg, for carrying out the measurements. The authors thank the Intel Corporation and the Fujitsu Group for providing their latest multicore hardware. Furthermore, the authors thank Miltenyi Biotec GmbH, Bergisch Gladbach, Germany and nanoPET Pharma $\mathrm{GmbH}$, Berlin, Germany, for providing their latest blood pool agents.

\footnotetext{
a) Author to whom correspondence should be addressed. Electronic mail: joscha.maier@dkfz.de; Telephone: +49(6221)423069; Fax: $+49(6221) 422585$.

${ }^{1}$ M. Salto-Tellez, S. Yung Lim, R. M. El-Oakley, T. P. L. Tang, Z. A. M. ALmsherqi, and S.-K. Lim, "Myocardial infarction in the C57BL/6J mouse: A quantifiable and highly reproducible experimental model," Cardiovasc. Pathol. 13, 91-97 (2004).

${ }^{2}$ S. Arber, J. J. Hunter, J. J. Ross, M. Hongo, G. Sansig, J. Borg, J.-C. Perriard, K. R. Chien, and P. Caroni, "MLP-deficient mice exhibit a disruption of cardiac cytoarchitectural organization, dilated cardiomyopathy, and heart failure," Cell 88, 393-403 (1997).

${ }^{3}$ J. J. Hunter, N. Tanaka, H. A. Rockman, J. J. Ross, and K. R. Chien, "Ventricular expression of a MLC- $2 \mathrm{v}$-ras fusion gene induces cardiac hypertrophy and selective diastolic dysfunction in transgenic mice," J. Biol. Chem. 270, 23173-23178 (1995).

${ }^{4}$ P. N. Costandi, L. R. Frank, A. D. McCulloch, and J. H. Omens, "Role of diastolic properties in the transition to failure in a mouse model of the cardiac dilatation," Am. J. Physiol. 291, H2971-H2979 (2006).

${ }^{5}$ T. G. Neilan, D. S. Jassal, T. M. Perez-Sanz, M. J. Raher, A. D. Pradhan, E. S. Buys, F. Ichinose, D. B. Bayne, E. F. Halpern, A. E. Weyman, G. Derumeaux, K. D. Bloch, M. H. Picard, and M. Scherrer-Crosbie, "Tissue Doppler imaging predicts left ventricular dysfunction and mortality in a murine model of cardiac injury," Eur. Heart J. 27, 1868-1875 (2006).
} 
${ }^{6}$ A. Saraste, V. Kytö, M. Saraste, T. Vuorinen, J. Hartiala, and P. Saukko, "Coronary flow reserve and heart failure in experimental coxsackievirus myocarditis: A transthoracic Doppler echocardiography study," Am. J. Physiol. 291, H871-H875 (2006).

${ }^{7}$ I. A. Sebag, M. D. Handschumacher, F. Ichinose, J. G. Morgan, R. Hataishi, A. C. T. Rodrigues, J. L. Guerrero, W. Steudel, M. J. Raher, E. F. Halpern, G. Derumeaux, K. D. Bloch, M. H. Picard, and M. Scherrer-Crosbie, "Quantitative assessment of regional myocardial function in mice by tissue Doppler imaging: Comparison with hemodynamics and sonomicrometry," Circulation 111, 2611-2616 (2005).

${ }^{8}$ J. E. Schneider, P. J. Cassidy, C. Lygate, D. J. Tyler, F. Wiesmann, S. M. Grieve, K. Hulbert, K. Clarke, and S. Neubauer, "Fast, high resolution in vivo cine magnetic resonance imaging in normal and failing mouse hearts on a vertical 11.7 T system," J. Magn. Reson. Imaging 18, 691-701 (2003).

${ }^{9}$ J. E. Schneider, F. Wiesmann, C. A. Lygate, and S. Neubauer, "How to perform an accurate assessment of cardiac function in mice using highresolution magnetic resonance imaging," J. Cardiovasc. Magn. Reson. 8, 693-693 (2006).

${ }^{10}$ W. D. Gilson, Z. Yang, B. A. French, and F. H. Epstein, "Measurement of myocardial mechanics in mice before and after infarction using multislice displacement-encoded MRI with 3D motion encoding," Am. J. Physiol. 288, H1491-H1497 (2005).

${ }^{11}$ B. Hiba, N. Richard, M. Janier, and P. Croisille, "Cardiac and respiratory double self-gated cine MRI in the mouse at 7 T," Magn. Reson. Med. 55, 506-513 (2006).

${ }^{12}$ C. T. Badea, B. Fubara, L. W. Hedlund, and G. A. Johnson, "4-D micro-CT of the mouse heart," Mol. Imaging 4, 110-116 (2005).

${ }^{13}$ S. A. Detombe, N. L. Ford, F. Xiang, X. Lu, Q. Feng, and M. Drangova, "Longitudinal follow-up of cardiac structure and functional changes in an infarct mouse model using retrospectively gated micro-computed tomography," Invest. Radiol. 43, 520-529 (2008).

${ }^{14}$ C. T. Badea, L. W. Hedlund, J. F. B. Mackel, L. Mao, H. A. Rockman, and G. A. Johnson, "Cardiac micro-computed tomography for morphological and functional phenotyping of muscle LIM protein null mice," Mol. Imaging 6, 261-268 (2007).

${ }^{15}$ M. Nahrendorf, C. T. Badea, L. W. Hedlund, J.-L. Figueiredo, D. E. Sosnovik, G. A. Johnson, and R. Weissleder, "High-resolution imaging of murine myocardial infarction with delayed-enhancement cine micro-CT," Am. J. Physiol. 292, H3172-H3178 (2007).

${ }^{16}$ C. T. Badea, L. W. Hedlund, and G. A. Johnson, "Micro-CT with respiratory and cardiac gating," Med. Phys. 31, 3324-3329 (2004).

${ }^{17}$ S. H. Bartling, W. Stiller, M. Grasruck, B. Schmidt, P. Peschke, W. Semmler, and F. Kiessling, "Retrospective motion gating in small animal CT of mice and rats," Invest. Radiol. 42, 704-714 (2007).

${ }^{18}$ N. L. Ford, H. N. Nikolov, C. J. D. Norley, M. M. Thornton, P. J. Foster, M. Drangova, and D. W. Holdsworth, "Prospective respiratory-gated micro-CT of free breathing rodents," Med. Phys. 32, 2888-2898 (2005).

${ }^{19}$ C. Badea, E. Bucholz, L. Hedlund, H. Rockman, and G. Johnson, "Imaging methods for morphological and functional phenotyping of the rodent heart," Toxicol. Pathol. 34, 111-117 (2006).

${ }^{20}$ N. L. Ford, M. M. Thornton, and D. W. Holdsworth, "Fundamental image quality limits for microcomputed tomography in small animals," Med. Phys. 30, 2869-2877 (2003).

${ }^{21}$ S. K. Carlson, K. L. Classic, C. E. Bender, and S. J. Russell, "Small animal absorbed radiation dose from serial micro-computed tomography imaging," Mol. Imaging Biol. 9, 78-82 (2007).

${ }^{22}$ S. Sawall, F. Bergner, R. Lapp, M. Mronz, M. Karolczak, A. Hess, and M. Kachelrieß, "Low-dose cardio-respiratory phase-correlated cone-beam micro-CT of small animals," Med. Phys. 38, 1416-1424 (2011).

${ }^{23}$ E. Y. Sidky and X. Pan, "Image reconstruction in circular cone-beam computed tomography by constrained, total-variation minimization," Phys. Med. Biol. 53, 4777-4807 (2008).

${ }^{24}$ X. Han, J. Bian, D. R. Eaker, T. L. Kline, E. Y. Sidky, E. L. Ritman, and X. Pan, "Algorithm-enabled low-dose micro-CT imaging," IEEE Trans. Med. Imaging 30, 606-620 (2011).
${ }^{25}$ L. Ritschl, S. Sawall, M. Knaup, A. Hess, and M. Kachelrieß, "Iterative 4D cardiac micro-CT image reconstruction using an adaptive spatio-temporal sparsity prior," Phys. Med. Biol. 57, 1517-1525 (2012).

${ }^{26}$ S. E. J. Armitage, S. I. Pollmann, S. A. Detombe, and M. Drangova, "Leasterror projection sorting to optimize retrospectively gated cardiac micro-CT of free-breathing mice," Med. Phys. 39, 1452-1461 (2012).

${ }^{27}$ C. T. Badea, S. M. Johnston, Y. Qi, and G. A. Johnson, "4D micro-CT for cardiac and perfusion applications with view under sampling," Phys. Med. Biol. 56, 3351-3369 (2011).

${ }^{28}$ C. T. Badea, E. Schreibmann, and T. Fox, "A registration based approach for 4D cardiac micro-CT using combined prospective and retrospective gating," Med. Phys. 35, 1170-1179 (2008).

${ }^{29}$ C. T. Badea, A. W. Wetzel, N. Mistry, S. Pomerantz, D. Nave, and G. A. Johnson, "Left ventricle volume measurements in cardiac micro-CT: The impact of radiation dose and contrast agent," Comput. Med. Imaging Graph. 32, 239-250 (2008).

${ }^{30}$ M. Kachelrieß, D.-A. Sennst, W. Maxlmoser, and W. A. Kalender, "Kymogram detection and kymogram-correlated image reconstruction from subsecond spiral computed tomography scans of the heart," Med. Phys. 29, 1489-1503 (2002).

${ }^{31}$ J. Kuntz, J. Dinkel, S. Zwick, T. Bäuerle, M. Grasruck, F. Kiessling, R. Gupta, W. Semmler, and S. H. Bartling, "Fully automated intrinsic respiratory and cardiac gating for small animal CT," Phys. Med. Biol. 55, 2069-2085 (2010).

${ }^{32}$ J. Dinkel, S. H. Bartling, J. Kuntz, M. Grasruck, A. Kopp-Schneider, M. Iwasaki, S. Dimmeler, R. Gupta, W. Semmler, H.-U. Kauczor, and F. Kiessling, "Intrinsic gating for small-animal computed tomography: A robust ECG-less paradigm for deriving cardiac phase information and functional imaging," Circulation 1, 235-243 (2008).

${ }^{33}$ L. A. Feldkamp, L. C. Davis, and J. W. Kress, "Practical cone-beam algorithm," J. Opt. Soc. Am. A 1, 612-619 (1984).

${ }^{34}$ G. C. Mc Kinnon and R. H. T. Bates, "Towards imaging the beating heart usefully with a conventional CT scanner," IEEE Trans. Biomed. Eng. BME-28, 123-127 (1981).

${ }^{35}$ K. L. Garden and R. A. Robb, "3-D reconstruction of the heart from few projections: A practical implementation of the McKinnon-Bates algorithm," IEEE Trans. Med. Imaging 5, 233-239 (1986).

${ }^{36}$ L. I. Rudin, S. Osher, and E. Fatemi, "Nonlinear total variation based noise removal algorithms," Physica D 60, 259-268 (1992).

${ }^{37}$ Z. Tian, X. Jia, K. Yuan, T. Pan, and S. B. Jiang, "Low-dose CT reconstruction via edge-preserving total variation regularization," Phys. Med. Biol. 56, 5949-5967 (2011).

${ }^{38}$ M. Persson, D. Bone, and H. Elmqvist, "Total variation norm for threedimensional iterative reconstruction in limited view angle tomography," Phys. Med. Biol. 46, 853-866 (2001).

${ }^{39}$ L. Ritschl, F. Bergner, C. Fleischmann, and M. Kachelrieß, "Improved total variation-based CT image reconstruction applied to clinical data," Phys. Med. Biol. 56, 1545-1561 (2011).

${ }^{40}$ H. M. Hudson and R. S. Larkin, "Accelerated image reconstruction using ordered subsets of projection data," IEEE Trans. Med. Imaging 13, 601609 (1994).

${ }^{41}$ N. Otsu, "A threshold selection method from gray-level histograms," IEEE Trans. Syst., Man, Cybern., Syst. 9, 62-66 (1979).

${ }^{42}$ P.-S. Liao, T.-S. Chen, and P.-C. Chung, "A fast algorithm for multilevel thresholding," J. Inf. Sci. Eng. 17, 713-727 (2001).

${ }^{43}$ R. Adams and L. Bischof, "Seeded region growing," IEEE Trans. Pattern Anal. Mach. Intell. 16, 641-647 (1994).

${ }^{44}$ S. Osher and J. A. Sethian, "Fronts propagating with curvature-dependent speed: Algorithms based on Hamilton-Jacobi formulations," J. Comput. Phys. 79, 12-49 (1988).

${ }^{45}$ T. F. Chan and L. A. Vese, "Active contours without edges," IEEE Trans. Image Process. 10, 266-277 (2001).

${ }^{46}$ Y. Shi and W. C. Karl, "A real-time algorithm for the approximation of level-set-based curve evolution," IEEE Trans. Image Process. 17, 645-656 (2008). 